\title{
ESTUDO DA INFLUENCIA DE PARÂMETROS RELEVANTES EM UM PROCESSO DE DESTILAÇÃO SOLAR DE ÁGUA SALGADA: MODELAGEM E SIMULAÇÃO
}

\author{
E. V. de FARIA ${ }^{1}$, N. G. SOUSA ${ }^{2}$ \\ ${ }^{1}$ Universidade Federal do Triângulo Mineiro, Instituto de Ciências Tecnológicas e Exatas \\ ${ }^{2}$ Universidade Federal do Triângulo Mineiro, Departamento de Engenharia Química \\ E-mail para contato: erica-victor@hotmail.com
}

\begin{abstract}
RESUMO - Um grande problema enfrentado atualmente pela sociedade é a diminuição na disponibilidade de água potável. Frente a este problema, se tornou evidente a necessidade de novas estratégias economicamente viáveis para que as águas salobras e marítimas também pudessem ser aproveitadas no consumo humano. Neste trabalho, foram investigados os efeitos da temperatura ambiente e velocidade do vento sobre um processo de destilação solar utilizando-se modelagem e simulação do processo no software $S c i l a b \AA$, e utilizando os dados meteorológicos da cidade de Uberlândia. Com isso foi possível verificar que estes dois parâmetros não exercem forte influência sobre a produção de água destilada, embora afetem diretamente a temperatura da cobertura e a temperatura da bacia.
\end{abstract}

\section{INTRODUÇÃO}

A escassez de água é um assunto cada vez mais recorrente. Apesar de ser um dos maiores bens da humanidade, a água não é inesgotável. De acordo com Cravo e Cardoso (1996), o consumo de água doce no mundo aumenta a um ritmo superior ao do crescimento populacional, restando, como uma das saídas para satisfazer às necessidades hídricas, a produção de água potável a partir da água do mar ou de águas salobras de açudes e poços.

Atualmente diversas tecnologias são utilizadas para a produção de água potável a partir de águas salgadas e salobras, dentre estas, a destilação solar se apresenta como uma alternativa atraente, uma vez que utiliza uma fonte de energia gratuita e abundante, de fácil operação, não polui e é eficaz no tratamento da água, sendo assim uma solução integrada para a escassez de água potável e problemas energéticos e ambientais enfrentados. O Brasil apresenta um elevado potencial para a utilização dessa fonte energética, já que possui altos índices anuais de radiação solar.

Em 1961 foram publicados dois importantes trabalhos na área de destilação solar o de Dunkle (1961) sobre destiladores solares simples e de múltiplo efeito e o trabalho de Lof et al. (1961) sobre balanço de energia em destiladores solares. A fim de propor uma comparação entre alguns tipos de destilação solar, Jorge (2011) estudou quatro tipos de instalações, testando a sensibilidade dessas estruturas à variação de alguns parâmetros. Hamadou (2014) estudou a modelagem e simulação de um sistema de destilação solar ativa com uso de um fluido de aquecimento abaixo da bacia d'água. 
O presente trabalho visa o estudo da dessalinização solar, pela investigação de seu modelo dinâmico e a avaliação da influência de variáveis relevantes como a temperatura ambiente e a velocidade do vento na produção diária de água destilada. Essa análise é baseada em estudos já feitos sobre dessalinização solar sendo assim possível avaliar a consistência dos resultados apresentados.

\section{DESCRIÇÃO DO PROCESSO}

A Figura 1 mostra uma unidade de destilação solar. Os objetivos em um processo de destilação é maximizar o calor gasto na evaporação da água da bacia ( $\left.Q_{\text {evaporação }}\right)$, pois esta é diretamente proporcional à produtividade do destilador e minimizar todas as formas de transferência de energia para o ambiente.

Figura 1 - Mecanismos de transferência de energia em um destilador solar passivo (adaptado de Duffie e Beckman (1991)).

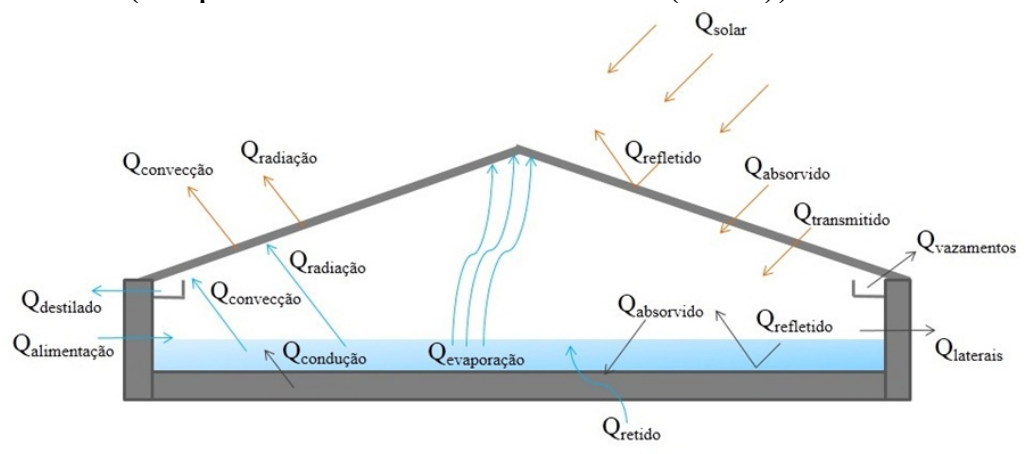

O modelo adotado para a simulação de um destilador solar é dado pelas Equações 1 a 3, estas são baseadas no modelo utilizado nos trabalhos de Jorge (2011) e Hamadou (2014). O modelo é composto por balanços de energia individuais para cada componente do destilador: a cobertura, base e água na bacia.

$$
\begin{aligned}
& \propto_{c} I_{s} A_{c}+h_{t w}\left(T_{w}-T_{c}\right) A_{b}=M_{c} C_{c} \frac{d T_{c}}{d t}+h_{1 g}\left(T_{c}-T_{a}\right) A_{c} \\
& \propto_{b}\left(1-\propto_{c}\right)\left(1-\propto_{w}\right) I_{s} A_{b}=h_{w}\left(T_{b}-T_{w}\right) A_{c}+h_{b}\left(T_{b}-T_{a}\right) A_{b} \\
& \alpha_{w}\left(1-\alpha_{c}\right) I_{s} A_{b}+h_{w}\left(T_{b}-T_{w}\right) A_{b}=M_{w} C_{w} \frac{d T_{w}}{d t}+h_{t w}\left(T_{w}-T_{c}\right) A_{b}
\end{aligned}
$$

em que: $T_{c}$ é a temperatura da cobertura, $T_{b}$ é a temperatura da bacia e $T_{w}$ é a temperatura da água da bacia.

O coeficiente total de transferência de calor da superfície da água para a cobertura é definido por: $h_{t w}=h_{r w}+h_{c w}+h_{e w}$. A transferência de calor por convecção ocorre a partir da mistura ar-vapor para a cobertura dado pela seguinte expressão: $h_{r w}=\varepsilon_{e f f} \sigma\left(T_{w}^{2}+T_{c}^{2}\right)\left(T_{w}+T_{c}\right)$, sendo $\sigma$ a constante de Boltzmann, a evaporação ocorre da bacia de água para a mistura arvapor dada por: $h_{c w}=0,884\left[T_{w}-T_{c}+\frac{\left(P_{w}-P_{c}\right) T_{w}}{268,9 * 10^{3}-P_{w}}\right]^{1 / 3}$ e a condensação ocorre da mistura 
ar-vapor para a cobertura dada pela equação: $h_{e w}=16,273 * 10^{-3} h_{c w} \frac{P_{w}-P_{c}}{T_{w}-T_{c}}$. As pressões parciais de vapor de água $\mathrm{P}_{\mathrm{w}}$ e $\mathrm{P}_{\mathrm{c}}$ em função da temperatura podem ser obtidas a partir da seguinte expressão: $P(T)=\exp \left[25,317-\frac{5144}{T}\right] . \quad$ O coeficiente de transferência de calor convectivo do revestimento da bacia para a água e o coeficiente total de transferência de calor do revestimento da bacia para o ambiente é dado pelas seguintes correlações, respectivamente: $\quad h_{b}=\left[\frac{L_{1}}{K_{1}}+\frac{1}{h_{1 g}}\right]^{-1}$ e $h_{w}=\frac{k_{w}}{L} 0,54(G r * P r)^{1 / 4}$, sendo o calor latente de vaporização da água do $\operatorname{mar}^{L}=3,146 * 10^{6}-2360 T_{w}$.

A velocidade do vento é determinada pela Equação 4, denominada de correlação de Mc Adams (Hamadou, 2014).

$$
h_{1 g}=\left\{\begin{array}{c}
5,6201+\frac{1151,2 V_{\text {vento }} ; \text { se } V_{\text {vento }}<4,88 \mathrm{~m} / \mathrm{s}}{T_{a}} \\
604,29\left(\frac{V_{\text {vento }}}{T_{a}}\right)^{0,78} ; \text { se } 4,88 \leq V_{\text {vento }} \leq 30,48 \mathrm{~m} / \mathrm{s}
\end{array}\right.
$$

A produção horária de água destilada é função da diferença entre a temperatura da cobertura e da água da bacia, dada por: $\dot{m}_{e w}=\frac{A_{c} h_{e w}\left(T_{w}-T_{c}\right) * 3600}{L}$.

destilado é dada pelo somatório da produção horária durante 0 tempr destilador solar: $M_{e w}=\sum_{i=1}^{24} \dot{m}_{e w}$.

\section{RESULTADOS E DISCUSSÕES}

Os dados de temperatura ambiente, radiação solar, velocidade do vento, adotados neste trabalho, são do mês de Janeiro de 2015 para cidade de Uberlândia-MG, todos estes dados foram obtidos pelo Instituto Nacional de Meteorologia (INMET). Os parâmetros utilizados na simulação estão dispostos na Tabela 1.

Tabela 1 - Parâmetros da simulação.

\begin{tabular}{c|c|c|c}
\hline $\begin{array}{c}\text { Absorbância da água } \\
\left(\alpha_{\mathrm{w})}\right.\end{array}$ & 0,08 & $\begin{array}{c}\text { Coeficiente de } \\
\text { dilatação térmica }(\beta)\end{array}$ & $0,000242761 / \mathrm{K}$ \\
\hline $\begin{array}{c}\text { Absorbância da base } \\
\left(\alpha_{\mathrm{b})}\right.\end{array}$ & 0,34 & $\begin{array}{c}\text { Condutividade térmica } \\
\text { da água }\left(\mathrm{k}_{\mathrm{w}}\right)\end{array}$ & $0,613 \mathrm{~W} / \mathrm{m}$ \\
\hline $\begin{array}{c}\text { Absorbância da } \\
\text { cobertura }\left(\alpha_{\mathrm{c})}\right.\end{array}$ & 0,05 & $\begin{array}{c}\text { Condutividade térmica } \\
\text { do isolamento }\left(\mathrm{k}_{1}\right)\end{array}$ & $0,08 \mathrm{~W} / \mathrm{m} \mathrm{K}$ \\
\hline Área da base $\left(\mathrm{A}_{\mathrm{b}}\right)$ & $1 \mathrm{~m}^{2}$ & $\begin{array}{c}\text { Emissividade da água } \\
\left(\varepsilon_{\mathrm{w}}\right)\end{array}$ & 0,96 \\
\hline Área da cobertura $\left(\mathrm{A}_{\mathrm{c}}\right)$ & $1,22 \mathrm{~m}^{2}$ & $\begin{array}{c}\text { Emissividade da } \\
\text { cobertura }\left(\varepsilon_{\mathrm{c}}\right)\end{array}$ & 0,9 \\
\hline
\end{tabular}




\begin{tabular}{c|c|c|c}
\hline $\begin{array}{c}\text { Capacidade calorifica } \\
\text { da água do mar }\left(\mathrm{C}_{\mathrm{w}}\right)\end{array}$ & $670 \mathrm{~J} / \mathrm{kg} \mathrm{K}$ & $\begin{array}{c}\text { Espessura do } \\
\text { isolamento }\left(\mathrm{L}_{1}\right)\end{array}$ & $0,06 \mathrm{~m}$ \\
\hline $\begin{array}{c}\text { Capacidade calorifica } \\
\text { da cobertura }\left(\mathrm{C}_{\mathrm{c}}\right)\end{array}$ & $4179 \mathrm{~J} / \mathrm{kg} \mathrm{K}$ & Massa de água & $10 \mathrm{~kg}$ \\
\hline
\end{tabular}

Os resultados obtidos nas simulações foram plotados na forma de gráficos para facilitar a visualização, como pode ser observado nas Figuras 2 e 3 . Ao observar o comportamento da temperatura ambiente, na Figura 2, pode-se notar que esta varia inversamente à variação da velocidade do vento, quanto maior a velocidade do vento, maior a temperatura observada. A mesma análise pode ser feita para a temperatura da água da bacia. Também se observa que o perfil de variação destas segue a mesma tendência. No entanto a produção de água destilada ao longo do dia não sofre mudanças significativas ao variar-se a velocidade do vento. Ainda, verifica-se que os resultados observados para o uso da velocidade do vento média nas simulações se aproximam dos mesmos para o uso da velocidade do vento com variação horária.

Figura 2: Comportamento da temperatura da cobertura, da água da bacia, e da produção de água ao longo do dia pra diferentes velocidades do vento mantendo a Temperatura ambiente fixa e igual a média diária $(\mathrm{Ta}=299,56 \mathrm{~K})(++$ Velocidade do vento variando ao longo do dia; -.- Velocidade do vento igual a $0 \mathrm{~m} / \mathrm{s}$; --- Velocidade do vento constante igual 1,62 m/s (média diária); $\cdots$ Velocidade do vento constante igual a $6 \mathrm{~m} / \mathrm{s}$ )
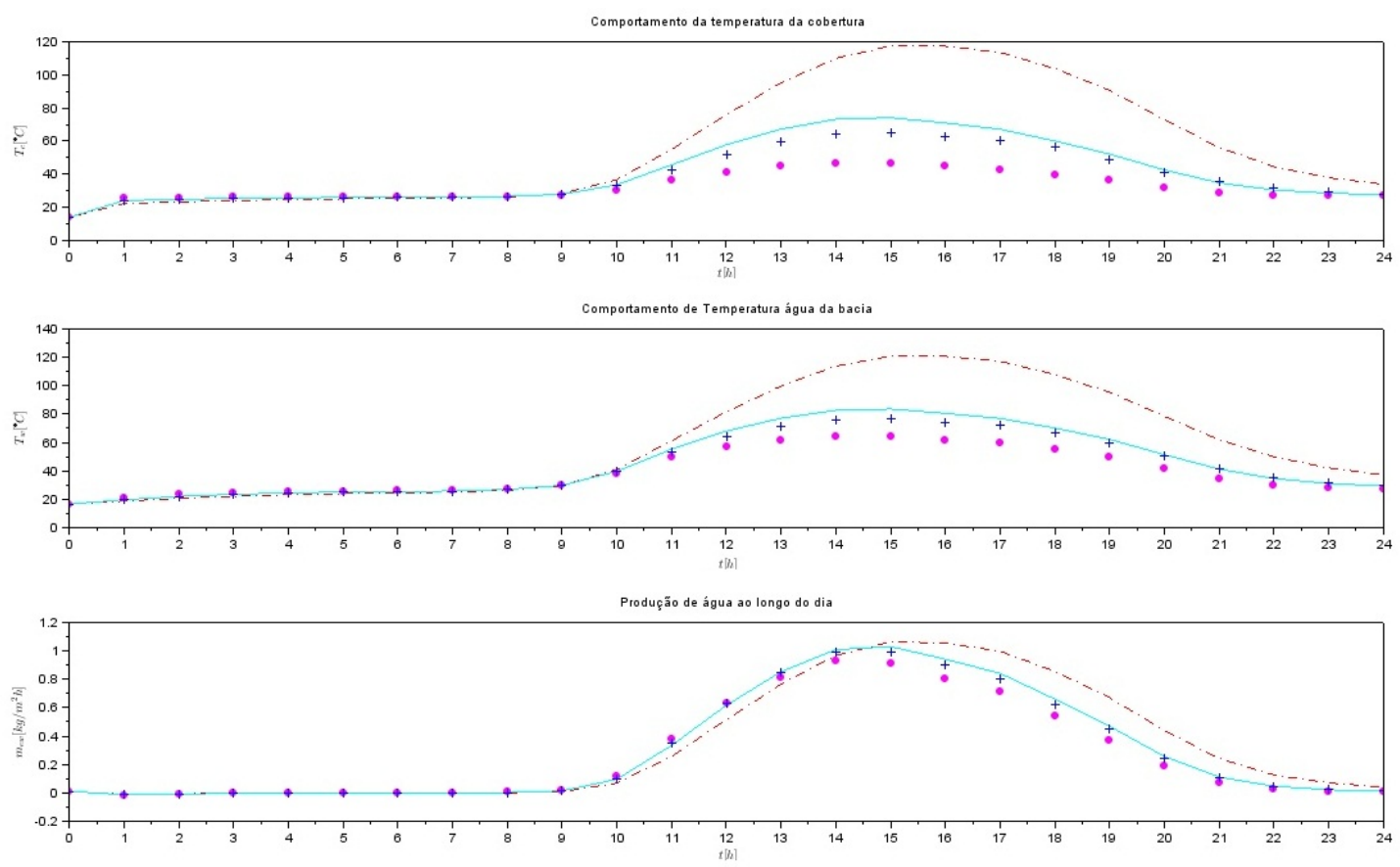

Para o segundo caso, Figura 3, em que se utilizou a temperatura ambiente horária, ou seja, variando ao longo do dia, têm-se as mesmas conclusões do primeiro caso. Também se observa que o comportamento das temperaturas e produção de água são semelhantes, o que leva a rematar que a variação da temperatura ambiente tem pouca influência sobre o processo 
de destilação solar e durante uma simulação optar pela utilização de uma temperatura média é uma boa aproximação do sistema real.

A produção total diária de água destilada em cada condição simulada está disposta na Tabela 2. Contudo, pode-se observar que a produção de água destilada sofre pouca influência da variável velocidade do vento. Sendo que ao desprezar este efeito a produção é acrescida em $12,6 \%$ do que ao considerar a velocidade do vento variando ao longo do dia. Também pode-se notar que a diferença na produção de água utilizando-se a temperatura ambiente real horária e a temperatura média é de aproximadamente $1,24 \%$.

Devido à grande influência que a velocidade do vento tem sobre a temperatura da cobertura era de se esperar que esta também tivesse forte influência sobre a produção. No entanto a força motriz do processo de destilação solar é o gradiente entre a temperatura da cobertura e da água da bacia, pois esta favorece a condensação do vapor de água, como é evidenciado pela equação produção horária de água destilada. Desta forma a pequena variação da produção diária é apoiada no fato de a velocidade do vento altera, de forma semelhante, essas suas variáveis, ou seja, em cada caso avaliado não há grandes alterações nesse gradiente. Tal resultado é concordante com o trabalho de Jorge (2011).

Figura 3:Comportamento da temperatura da cobertura, da água da bacia, e da produção de água ao longo do dia para diferentes velocidades do vento utilizando a temperatura ambiente horária. (++ Velocidade do vento variando ao longo do dia; -.- Velocidade do vento igual a 0 $\mathrm{m} / \mathrm{s}$; -- Velocidade do vento constante igual 1,62 m/s (média diária);

$\cdots$ Velocidade do vento constante igual a $6 \mathrm{~m} / \mathrm{s}$ )
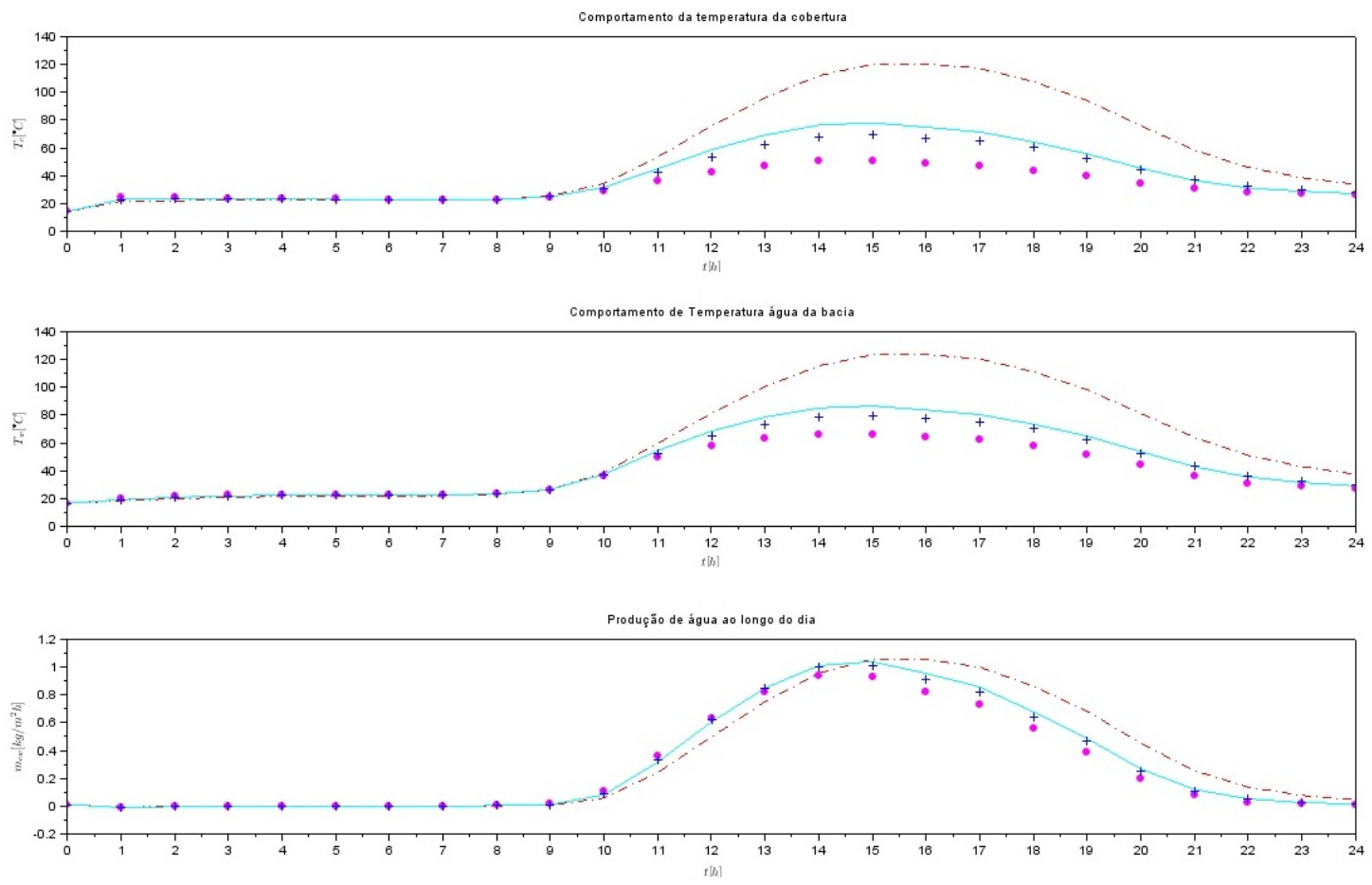

Tabela 2: Produção diária de água para cada condição

\begin{tabular}{|l|l}
$\mathbf{T}_{\mathrm{A}}$ constante & $\mathbf{T}_{\mathrm{A}}$ horária
\end{tabular}




\begin{tabular}{c|c|c|c}
\hline $\mathbf{V}_{\text {vento }}$ real horária & $7,104 \mathrm{~kg} / \mathrm{m}^{2}$ dia & $\mathbf{V}_{\text {vento }}$ real horária & $7,193 \mathrm{~kg} / \mathrm{m}^{2}$ dia \\
\hline $\mathbf{V}_{\text {vento }}=\mathbf{0} \mathbf{~ m} / \mathbf{s}$ & $8,126 \mathrm{~kg} / \mathrm{m}^{2}$ dia & $\mathbf{V}_{\text {vento }}=\mathbf{0 ~ m} / \mathbf{s}$ & $8,129 \mathrm{~kg} / \mathrm{m}^{2}$ dia \\
\hline $\mathbf{V}_{\text {vento }}=\mathbf{1 , 6 2} \mathbf{~ m} / \mathbf{s}$ & $7,290 \mathrm{~kg} / \mathrm{m}^{2}$ dia & $\mathbf{V}_{\text {vento }}=\mathbf{1 , 6 2} \mathbf{~ m} / \mathbf{s}$ & $7,360 \mathrm{~kg} / \mathrm{m}^{2}$ dia \\
\hline $\mathbf{V}_{\text {vento }}=\mathbf{6 ~ m} / \mathbf{s}$ & $6,469 \mathrm{~kg} / \mathrm{m}^{2}$ dia & $\mathbf{V}_{\text {vento }}=\mathbf{6 ~ m} / \mathbf{s}$ & $6,589 \mathrm{~kg} / \mathrm{m}^{2}$ dia \\
\hline
\end{tabular}

\section{CONCLUSÕES}

Através da análise e simulação de um sistema de destilação solar foi estudada a influência de se considerar a temperatura ambiente como a média diária em contrapartida com o uso da temperatura real horária, ou seja, variando ao longo do dia. Neste caso pôde-se notar que a adoção de uma temperatura média diária é uma boa aproximação durante a simulação de sistemas de destilação solar de água salgada.

Outro ponto avaliado foi a influência da velocidade do vento utilizando-se de 3 níveis de comparação, mantendo-a constante e variando ao longo do dia. Novamente pôde-se notar que vento não exerce alteração considerável sobre a produção do destilado, visto que este parâmetro exerce influência direta e de igual grandeza sobre a temperatura da cobertura e da água, e por isso não influi em grandes variações no gradiente de temperatura dentro do destilador solar e com isso na produção de destilado. Portanto, assim como verificado no trabalho de Jorge (2011), pode-se afirmar que a velocidade do vento não é uma variável de forte influência sobre a destilação solar.

\section{REFERÊNCIAS}

CRAVO, J. G.; CARDOSO, H. E. Projeto de dessalinização de solos e água. Nota Técnica $\mathrm{n}^{\circ} 1$. Brasília/DF: SRH/MMA, 1996.

DUfFIE, J., \& BECKMAN, W. Solar Engineering of Thermal Processes (2. ${ }^{\text {a }}$ ed.). New York: John Wiley and Sons, 1991.

DUNKLE, R. V. Solar water distillation: the roof type still and a multiple effect

Instituto Nacional de Meteorologia (INMET) Disponível em: $<$ http://www.inmet.gov.br/portal/>.

JORGE, Bruno Miguel Jacinto: Simulação de Processos de Destilação Solar de Água Salgada. Faculdade de Engenharia Mecânica. Universidade Técnica de Lisboa, Outubro de 2011. 118p. Dissertação de Mestrado.

LOF, G. O. G., EIBLING, J. A., BLOEMER, J. M. Energy balances in Solar Distillers. A.I.Ch.E. Journal, vol. 7, $\mathrm{N}^{\circ}$ 4, 641-649, 1961. 